\title{
The pain, the oncologist
}

\author{
Sebastiano Mercadante
}

Received: 14 May 2014 / Accepted: 26 May 2014 / Published online: 6 June 2014

(C) Springer-Verlag Berlin Heidelberg 2014

In this issue of Supportive Care in Cancer, new data regarding the epidemiology of cancer pain and breakthrough pain (BTP) in an oncologic population are reported [1]. The editorial process was complex due to contrasting opinions among reviewers. The main problem was related to the unselected cohort of cancer population, independently from the stage of disease, admitted to an outpatient oncology clinic; crosssectional design; reporting of old data; no distinction between new patients and follow-up visits; or lack of definition for palliative care patients and patients visited for oncologic treatments. Moreover, some issues, like the pain interferences with sleep, cannot be appropriately assessed, unless in longitudinal studies, providing only a mere association rather than a clear relationship. Finally, it is unclear which one of the pains, background pain or breakthrough pain, is interfering with sleep. While this kind of study offers a photograph of an institutional population referred to an outpatient oncologic clinic, data cannot be extended to other settings or other population. Perhaps, a comparison with a similar oncological population reported in large sample of patients existing in literature would have been of benefit $[2]$.

Nevertheless, these data regarding this unselected population attending an oncologic outpatient clinic reflect what may happen in this context and provide useful information. Oncologists should routinely screen

S. Mercadante $(\bowtie)$

Anesthesia \& intensive Care \& Pain Relief and Supportive Care, La Maddalena Cancer Center, Via San Lorenzo 312, 90146 Palermo, Italy

e-mail: 03sebelle@gmail.com

S. Mercadante

e-mail: terapiadeldolore@lamaddalenanet.it patients with pain, and focus on its characteristics to plan an adequate treatment. An early identification of patients to be referred to a palliative care consultation is of paramount importance [3]. On the other hand, a positive cultural pressure of palliative care teams may positively influence the attitudes of oncologists in cancer pain management [4]. Authors report that $22 \%$ of patients have a significant pain, that I assume to be $>4$, but overall, the mean intensity was quite low. Unfortunately, we do not know if it is the outcome of an analgesic treatment or a spontaneous finding. Data regarding the appropriateness of the use of analgesics would have been of benefit. From these data, it seems that patients are in a very early phase of disease, metastatic or not, as only a minority potentially would require some therapeutic intervention at those levels of pain intensity. Alternatively, one could argue that oncologists are treating relatively well their patients.

Breakthrough pain has been the subject of several controversies, either from the epidemiological or therapeutical point of view. It is unclear if the denominator of $21 \%$ found in this study regards the entire population examined or the number of patients with chronic pain. Given the lack of a clear definition "a priori", we are unable to interpret this information. The prevalence of breakthrough pain has been recently assessed in a pooled analysis of literature [5]. The lowest prevalence rates were detected in studies conducted in outpatient clinics (39.9\%), and the highest prevalence was reported in studies conducted in hospice $(80.5 \%)$. It has been established that the large variability depends on the setting, as well as the clinical and organizational issues. For instance, clear differences have been found between very advanced cancer patients followed at home and unselected patients recruited in an oncologic setting, like those observed in this study [6, 
7]. Thus, breakthrough pain should be considered as a dynamic entity dependent on several factors, including analgesic treatment, level of physical activity, and the course of disease [8].

Conflict of interest No conflicts to declare

\section{References}

1. Raj SX, Thronaes M, Brunelli C, Hjermstad MJ, Klepstad P, Kaasa S (2014) A cross-sectional study on prevalence of pain and breakthrough pain among an unselected group of outpatients in a tertiary cancer clinic. Support Care Cancer (Epub ahead of print)

2. Mercadante S, Roila F, Berretto O, Labianca R, Casilini S, DOMAINAIOM study group (2008) Prevalence and treatment of cancer pain in Italian oncological wards centres: a cross-sectional survey. Support Care Cancer 16:1203-1211
3. Kwon JH, Hui D, Chisholm G, Ha C, Yennurajalingam S, Kang JH, Bruera E (2013) Clinical characteristics of cancer patients referred early to supportive and palliative care. J Palliat Med 16:148-155

4. Mercadante S, Guccione C, Di Fatta S et al (2013) Cancer pain management in an oncological ward in a comprehensive cancer center with an established palliative care unit. Support Care Cancer 21:3287-3292

5. Deandrea S, Corli O, Consonni D et al (2014) Prevalence of breakthrough cancer pain: a systematic review and a pooled analysis of published literature. J Pain Symptom Manag 47:57-76

6. Mercadante S, Costanzo BV, Fusco F et al (2009) Breakthrough pain in advanced cancer patients followed at home: a longitudinal study. $\mathrm{J}$ Pain Symptom Manag 38:554-560

7. Mercadante S, Zagonel V, Breda E et al (2010) Breakthrough pain in oncology: a longitudinal study. J Pain Symptom Manag 40:183-190

8. Mercadante S, Valle A, Porzio G, Aielli F, Adile C, Ficorella C, Raineri M, Giarratano A, Casuccio A (2013) Relationship between background cancer pain, breakthrough pain, and analgesic treatment: a preliminary study for a better interpretation of epidemiological and clinical studies. Curr Med Res Opin 29: $667-671$ 